\section{CONTABILIDAD POR ÁREAS DE RESPONSABILIDAD}

\section{Dr. ERNESTO POLAR FALCÓN}

La notable evolución del mundo actual de los negocios ha obligado a las empresas a revisar constantemente sus técnicas de administración y de contabilidad, para que éstas aporten a sus directivos, elementos suficientes y oportunos para tomar decisiones.

En México, desde hace un tiempo atrás, el Instituto Mexicano de Contadores Públicos A.C., recomienda a los contadores públicos y a los hombres de empresas, el uso del sistema de Contabilidad por Áreas de Responsabilidad, que tiene como propósito identificar los presupuestos de operación y los resultados obtenidos en la empresa, con los funcionarios responsables de su ejecución, con el objeto de que puedan localizarse con mayor facilidad las áreas en donde se encuentran los problemas y las personas que responden a los mismos. Este sistema de información es un elemento utilísimo para «administrar por excepción» y lograr que el personal ejecutivo se sienta parte del cuerpo que dirige los negocios de las empresas.

La Universidad Nacional Mayor de San Marcos, por intermedio de la revista Quipukamavoc, vocero del Instituto de Investigación de Ciencias Financieras y Contables de la Facultad de Ciencias Contables, considera propicia la ocasión, para incentivar a sus docentes, a sus alumnos y en general a todos los contadores públicos, a la investigación de este interesante sistema de información, que indudablemente traería óptimos resultados, para tomar decisiones oportunas en el desarrollo de las operaciones de la empresa peruana, hoy en día, duramente maltratada por la recesión.

Para una mejor comprensión del sistema de Contabilidad por Áreas de Responsabilidad. a continuación se analizan sus conceptos principales:

1. Tradicionalmente, la contabilidad administrativa, que es la que se aplica en el Perú, clasifica los gastos de acuerdo con su naturaleza (sueldos, depreciación, alquiler, etc.), y la contabilidad de costos de producción utiliza centros o departamentos que le sirven como puntos convenientes para acumular gastos, consumos y provisiones, como paso previo para aplicarlos al costo de los productos obtenidos.

La Contabilidad por Áreas de Responsabilidad cambia este enfoque, para identificar las operaciones: gastos, consumos y provisiones, con los ejecutivos responsables de dichas operaciones.

2. La Contabilidad por Áreas de Responsabilidad no se conforma con identificar las operaciones con el ejecutivo responsable, sino que utiliza medidas de comparación. generalmente presupuestos y costos estándar, para medir resultados.

3. La información operativa, que tradicionalmente se entrega a la gerencia general y a otros altos ejecutivos, ahora se lleva, debidamente seleccionada, a todas las áreas involucradas y para las cuales se ha establecido responsabilidad de supervisión.

La Contabilidad por Áreas de Responsabilidad, haciendo uso de todos los elementos que se han señalado, se 
transforma en un plan de administración. Efectivamente, se intenta crear conciencia de administración en todas las personas responsables de las operaciones, haciendo que ellos intervengan directamente en la formulación de sus objetivos, que comparen esos objetivos con los resultados realmente obtenidos, que expliquen las variaciones, y que den sugerencias que en su opinión procedan a mejorar la marcha de la empresa. Sobre esta base, se crea pues «un cuerpo administrativo» valiosísimo para la gerencia general de la empresa.

Se ha dicho que la Contabilidad por Áreas de Responsabilidad, identifica los gastos e ingresos con los responsables y posteriormente va a medir los resultados obtenidos por cada uno de ellos. Pues bien, para poder lograr esto, es indispensable que la empresa tenga claramente definidas las funciones y responsabilidades de cada uno de sus departamentos y de su personal. Debe contar. por lo tanto, con una gráfica de organización que corresponda a la realidad y en donde se muestren, con claridad. estas funciones y responsabilidades: no se intente establecer una Contabilidad por Áreas de Responsabilidad si previamente no se ha definido la responsabilidad de cada área.

Normalmente, en la Contabilidad por Áreas de Responsabilidad, se debe establecer el control operativo y financiero en cuatro niveles:

1. El gerente general, en el primer nivel, quien es responsable ante el directorio de la empresa, de la utilidad obtenida en un período económico.

2. Los gerentes de divisiones tradicionales: compra, producción, ventas y finanzas; en el segundo nivel. responsables de las operaciones de sus propias áreas, ante el gerente general.

3. Los jefes de departamentos en el tercer nivel, responsables ante el gerente de una división.

4. Y el cuarto nivel, constituido por los supervisores de las diferentes secciones que conforman un departamento. responsables ante el jefe de un departamento.

En general, no se considera práctico bajar más allá del cuarto nivel que se ha precisado.

Ahora bien, una vez que cada función desempeñada por la empresa esté asignada a alguna unidad de la organización; que la asignación de responsabilidad sea específica y se entienda; y que cada posición de la organización informe a un solo jefe, se puede afirmar que se tiene listo el recipiente en el cual se va a colocar todo el sistema de la empresa.

Es importante preparar un nuevo Plan de Cuentas, que facilite la clasificación de los gastos, costos e ingresos en la misma forma que se tienen clasificadas las responsabilidades en el nuevo organigrama de la empresa. El objetivo del Plan de Cuentas es precisamente establecer las cuentas y subcuentas necesarias para agrupar las operaciones, de tal manera que al finalizar cada mes, se pueda informar a cada responsable, sobre los gastos, costos e ingresos de su área, de dicho pelíodo operativo.

Pero al hablar de una Contabilidad por Áreas de Responsabilidad. no se la puede limitar solamente a la simple aplicación de datos históricos hacia las áreas involucradas; es indispensable que se comparen estos datos. con lo que realmente ha ocurrido, con las medidas de eficiencia 


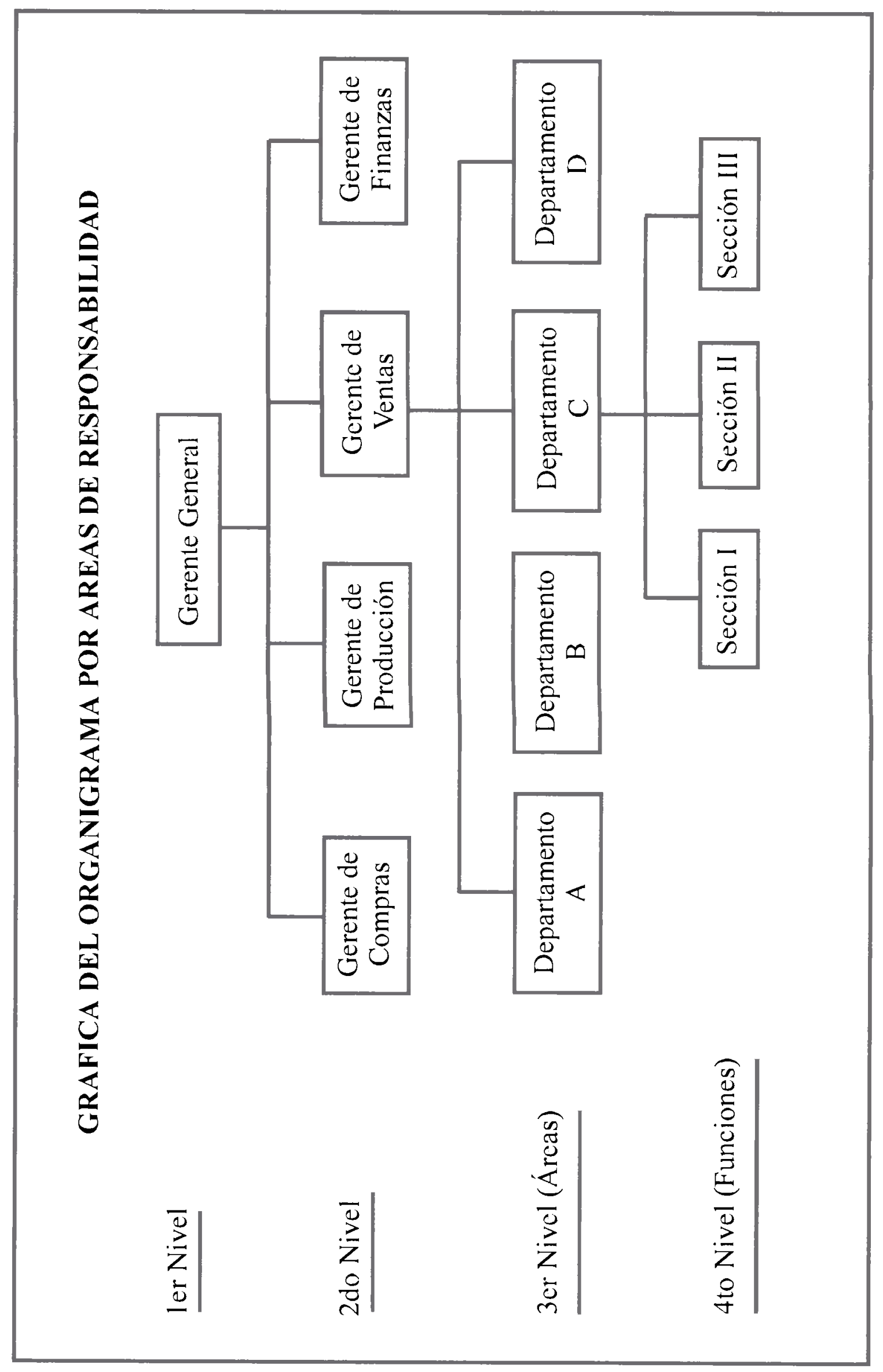


que previamente se hayan establecido y que llevan el propósito de conocer lo que debió haber sucedido, en condiciones normales de eficiencia. Es en esta forma. cómo se puede conocer la eficiencia o la ineficiencia con la que trabajan los ejecutivos de la empresa, los departamentos y secciones que la forman y la organización general.

Las medidas de eficiencia que se utilicen pueden ser muy diversas; pero siempre debe tenerse en cuenta que de utilizar alguna que no sea apropiada, se corre el riesgo de tomar decisiones indebidas.

Es por eso que se recomienda el control presupuestal y la aplicación de los costos estándar, elementos técnicos que son ampliamente conocidos y que han probado su confiabilidad. cuando son utilizados por personal idóneo.

\section{Para que la Contabilidad por Áreas}

de Responsabilidad, sea verdaderamente un instrumento contable que controle adecuadamente, debe auxiliarse de una técnica presupuestal, debidamente depurada y que incluya por lo menos lo siguiente:

1. Los presupuestos deben plasmar las políticas más importantes de la empresa, debidamente estudiadas por la gerencia general y la plana ejecutiva, contando asimismo con la aprobación del directorio. Este paquete de políticas más importante será el punto central que coordine los presupuestos que preparen, posteriormente. cada uno de los departamentos de la empresa.

2. En la elaboración de los presupuestos, deben intervenir todos los departamentos involucrados, en los niveles que pretenda controlar la Contabilidad por Áreas de Responsabilidad. recomendando que estos niveles sean cuatro. Los ejecutivos, funcionarios y empleados que intervengan en la evolución del presupuesto, bajo la coordinación del gerente de presupuestos, deben estar conscientes de su función. de la responsabilidad que absorben y de la forma en que se coordina su presupuesto parcial con el presupuesto general.

3. Es conveniente que la información fluya de abajo hacia arriba; en otras palabras, cada gerente, jefe o supervisor, tomando en cuenta los objetivos generales que se pretenden en la empresa y en su propia área, debe fijar sus propios objetivos particulares; estos objetivos, a su vez. plasmados en un presupuesto, serán juzgados, revisados y en su caso modificados, después de escuchar la opinión del responsable de dicho presupuesto, por el superior inmediato, hasta llegar a la última instancia, en este caso, al gerente general, responsable del presupuesto final de la empresa.

En esta forma, se logrará que los ejecutivos de la empresa, participen en la administración de la misma; que se forme en ellos, la conciencia de control de costos y obtención de objetivos tan indispensables para lograr los fines de la Contabilidad por Áreas de Responsabilidad. Se pretende con esto, no sólo tener un magnífico administrador en la persona del gerente general, sino haber formado un equipo administrativo en cuatro niveles diferentes, que auxilie en sus decisiones al gerente general, y que tenga como meta y como orgullo el logro de objetivos que ellos mismos se han fijado y que se sientan parte interesada del progreso de la empresa.

4. Los presupuestos deben ser, en todo momento, realistas. No deben forzarse sus proyecciones, en tal forma que al compararlas con los saldos reales, se sufran decepciones que perjudiquen el ánimo de los ejecutivos y en consecuencia, la buena marcha de la empresa. 
5. Es recomendable el uso de presupuestos exibles. debidamente coordinados con la

ntabilidad por Areas de Responsabilidad.

El Control Presupuestal es uno de los mejores instrumentos que se tiene para vigilar la buena marcha de la empresa; pero en el área de producción, conviene complementarlo con la aplicación de los costos estándar, para contar con un elemento más, y es esta área con mayor razón, para conocer la eficiencia de su trabajo.

Efectivamente, en esta época. es frecuente que el gerente general y la plana mayor de ejecutivos, ya no se conforme con conocer el costo a que resultó un determinado producto. sino que estén interesados en conocer también el costo a que debió resultar ese producto; y para proporcionar esta información, nada mejor que la aplicación de los costos estándar, que se constituyen en patrones de eficiencia y que, a través del análisis de sus variaciones, permiten conocer la forma en que están trabajando los diversos departamentos de producción y precisar responsabilidades.

Los costos estándar, como se señaló antes para los presupuestos. deben ser realistas; en otras palabras, deben pretender

GRAFICA DEL SISTEMA DE COSTOS ESTANDAR

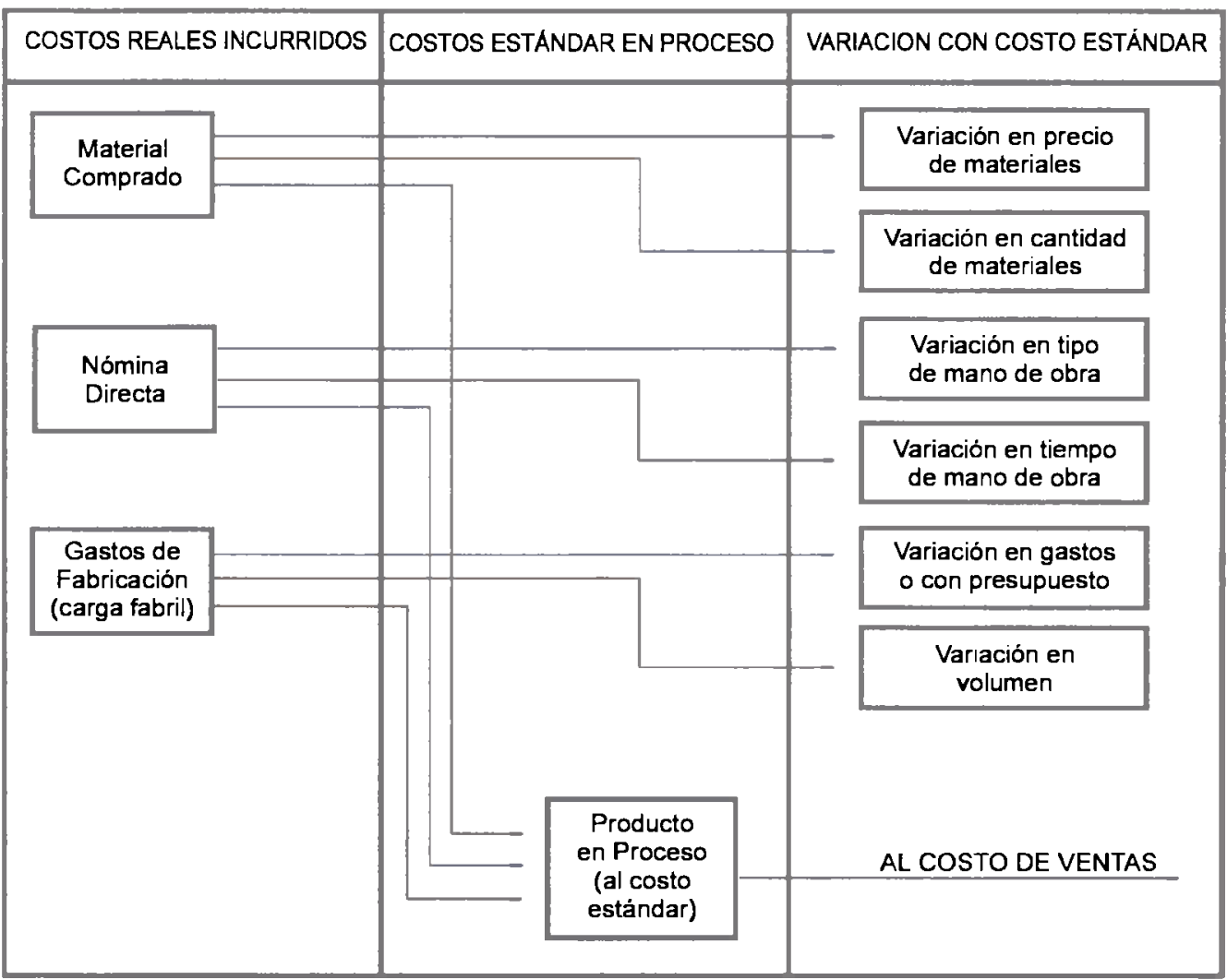


señalar el costo a que debería lograrse un producto, a un volumen de producción determinado y en condiciones operativas en las que se supone una eficiencia que pueda alcanzar la empresa con los elementos que tiene disponibles.

Para que los costos estándar sean operantes, como medida de eficiencia, deben ser producto de una investigación detenida hecha por expertos en la materia, más bien, que el resultado de cálculos hechos por la contabilidad.

Es peligroso hacer comparaciones de datos reales con patrones de eficiencia de los costos estándar, que no estén debidamente estudiados y tomar decisiones y exigir responsabilidades en base a las variaciones que resulten.

Con todo lo expuesto, se puede establecer una organización en la que se tienen definidas las líneas de autoridad y las funciones y responsabilidades de cada área; preparar un control presupuestal, en cuya determinación de objetivos han intervenido directamente y en forma muy especial, todos los ejecutivos y funcionarios que tienen a su cargo la supervisión de una función; y además, se cuenta con los costos estándar, elaborados técnicamente, que permiten medir la eficiencia lograda en los departamentos de producción.

Con estos instrumentos, se logra determinar periódicamente, las desviaciones entre los datos reales y las medidas de eficiencia establecidas; $y$ con estas variaciones, se puede identificar el departamento y el ejecutivo o funcionario responsable. Es aquí, cuando se logra una de las etapas más interesantes de la Contabilidad por Áreas de Responsabilidad.

Teniendo el sistema planeado en esta forma, es posible aplicar los principios de la administración por excepción.

Efectivamente, los presupuestos y los costos estándar, constituyen medidas de eficiencia, que en forma realista se pretenden alcanzar. Quiere decir, que todas aquellas operaciones que marchen de acuerdo con dichos presupuestos y los costos estándar, están dentro de los márgenes de eficiencia, previstos con anticipación. Entonces, la atención debe concentrarse, en forma muy particular, hacia las desviaciones a los planes trazados. Es aquí, en donde se pone el mayor esfuerzo para conocer los problemas que originaron las deficiencias; para definir alternativas para solucionarlos, para tomar acción que implique correcciones inmediatas o planes de trabajo a largo plazo.

Es pues menester, aprovechar el tiempo de los altos ejecutivos de la empresa, en el análisis detenido de los problemas detectados, en vez de desviar su atención hacia un sinnúmero de operaciones que están dentro de los márgenes de eficiencia, que ellos mismos consideran razonables.

\section{Por otro lado, la Contabilidad por} Áreas de Responsabilidad debe coordinarse tanto con la contabilidad general, como con la contabilidad de costos industriales. Las tres, por lo que toca a las cuentas de resultados, persiguen objetivos que pueden complementarse adecuadamente. La contabilidad general agrupa las operaciones en forma tal. que los ingresos, costos y gastos queden debidamente clasificados de acuerdo con su naturaleza; la contabilidad de costos industriales, por su parte, lleva como objetivo la aplicación hacia los centros de costos de los gastos y consumos incurridos en los departamentos de producción y de las provisiones que los afecten. con la finalidad de aplicar estos costos hacia los productos y determinar los costos unitarios de fabricación. 


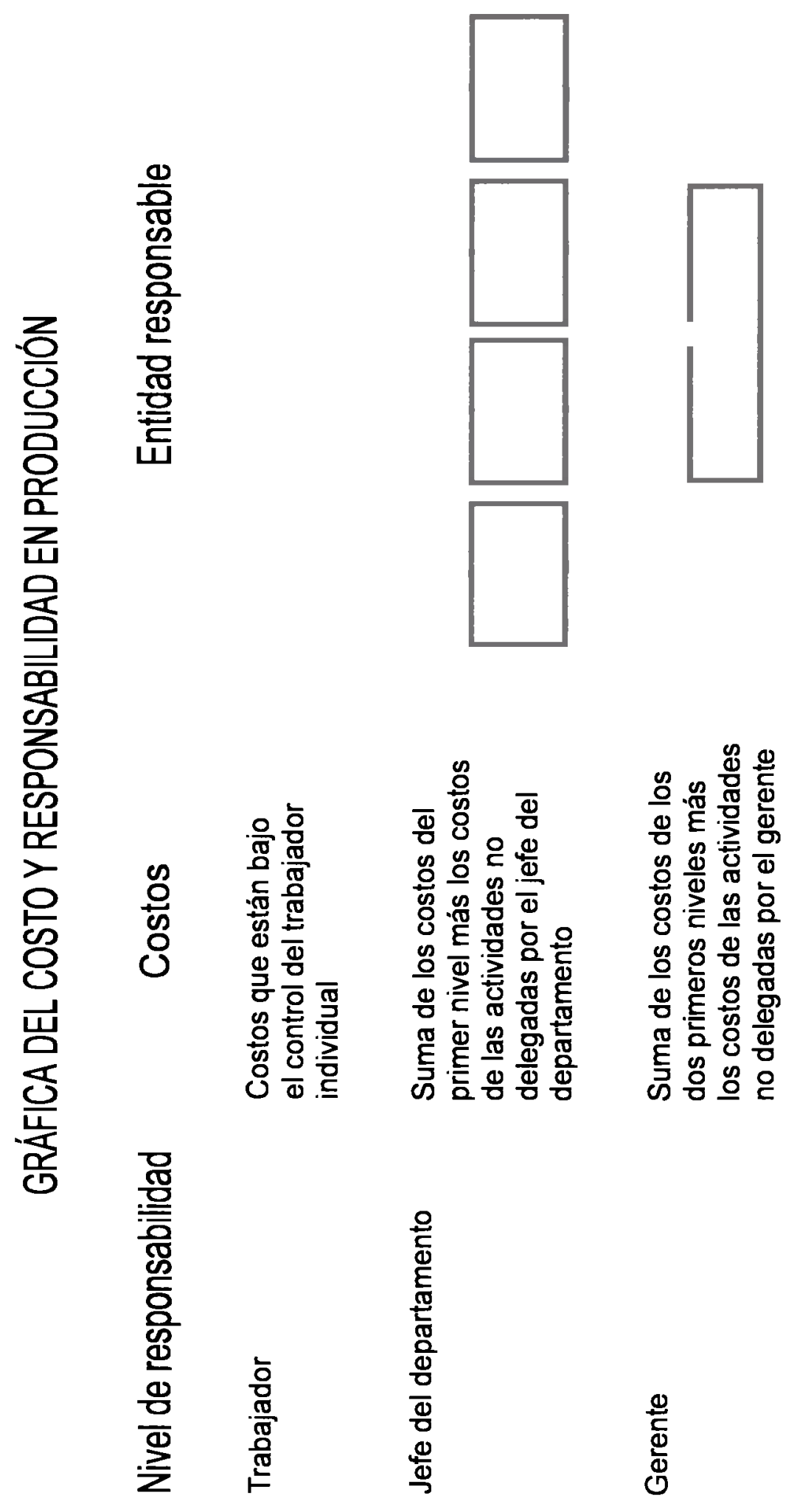


La Contabilidad por Áreas de Responsabilidad identifica el gasto, el costo y el ingreso con los departamentos y ejecutivos y funcionarios responsables; es decir, las mismas partidas que se registran en la contabilidad general y en la contabilidad de costos industriales, pasan a formar parte de la Contabilidad por Áreas de Responsabilidad. pero con un enfoque diferente.

Ningún sistema, por bueno que sea, puede trabajar de manera efectiva, sin buena comunicación. No es raro encontrar en la práctica, trabajos bien desempeñados y con buenas conclusiones, que son archivados en la gerencia general. por la sencilla razón de que el informe fue presentado en forma inadecuada o al ejecutivo que no es el apropiado.

\section{En la Contabilidad por Áreas de} Responsabilidad. la información es básica. Si verdaderamente se quiere formar un equipo administrativo que tenga conciencia y entusiasmo para alcanzar sus objetivos, por reducir costos y gastos. por generar los ingresos previstos, etc., se tiene que dar a los integrantes del equipo administrativo, información amplia y oportuna sobre sus logros

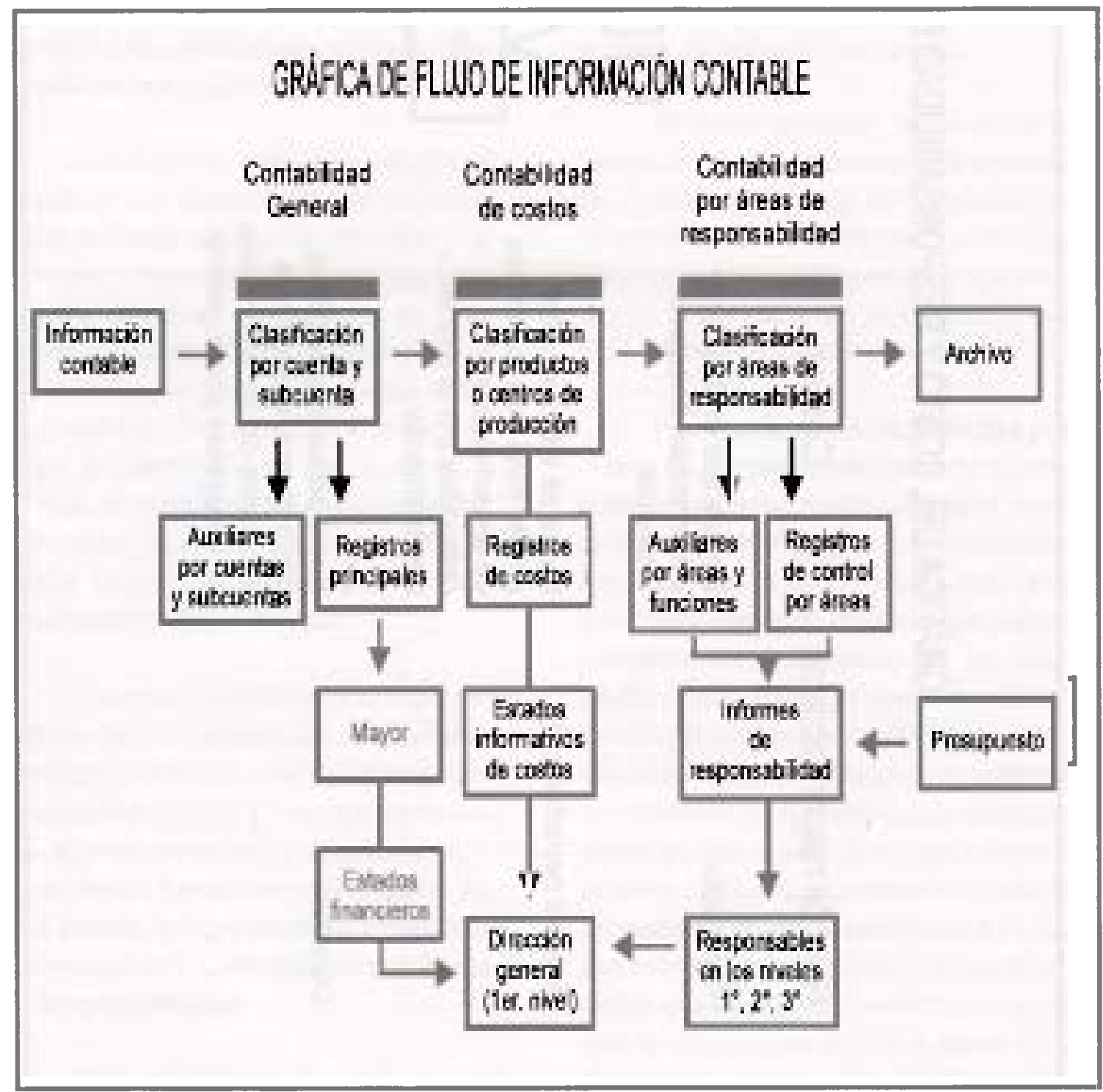


sobre sus deficiencias. Sólo así, la Contabilidad por Áreas de Responsabilidad. puede lograr sus objetivos.

Los responsables deben recibir información específica sobre su área, debidamente comparada con los índices de eficiencia u objetivos que les han fijado. La información debe presentarse en forma sencilla y legible, destacando lo que verdaderamente tiene importancia y eliminando lo que desvía innecesariamente la atención. Conviene tomar en cuenta que el uso de gráficas normalmente facilita la interpretación de los resultados y muestra tendencias que ayudan a la toma de decisiones.

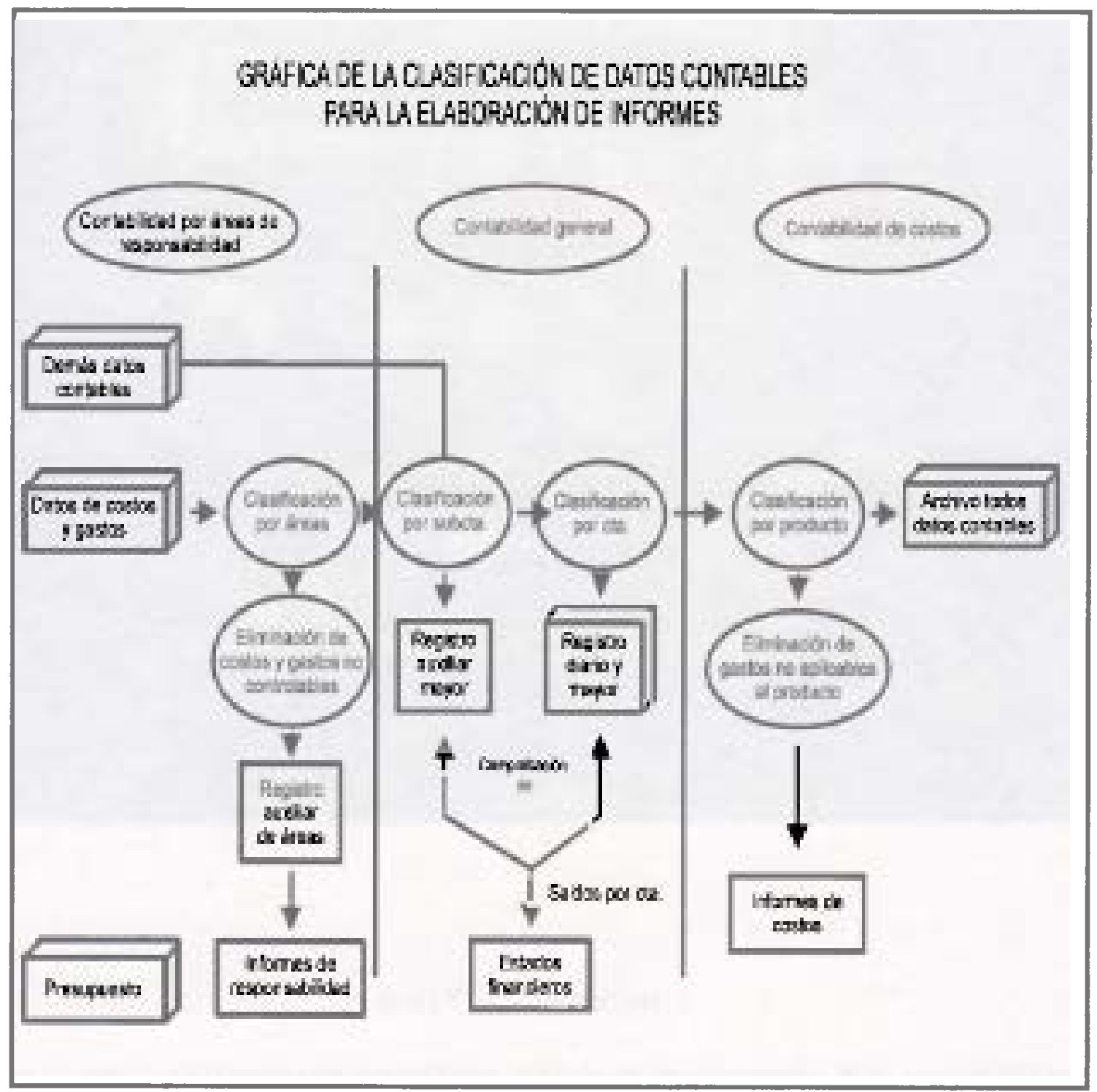

